\title{
Multivariable Model Reference Adaptive Control of an Industrial Power Boiler Using Recurrent RBFN
}

\author{
Jafar Tavoosi $\left(\mathbb{D},{ }^{1}\right.$ Yavar Azarakhsh $\left(\mathbb{D},{ }^{1}\right.$ Ardashir Mohammadzadeh $\left(\mathbb{D},{ }^{2}\right.$ Saleh Mobayen (iD), \\ Jihad H. Asad ${ }^{1},{ }^{4}$ and Rabia Safdar ${ }^{5,6}$ \\ ${ }^{1}$ Department of Electrical Engineering, Faculty of Engineering, Ilam University, Ilam, Iran \\ ${ }^{2}$ Department of Electrical Engineering, University of Bonab, Bonab, Iran \\ ${ }^{3}$ Future Technology Research Center, National Yunlin University of Science and Technology, Douliu, Yunlin 64002, Taiwan \\ ${ }^{4}$ Department of Physics, Faculty of Applied Sciences, Palestine Technical University, P. O. Box 7, Tulkarm, State of Palestine \\ ${ }^{5}$ Department of Mathematics, Lahore College Women University, Lahore, Pakistan \\ ${ }^{6}$ Department of Mathematics, College of Arts and Sciences, University of Jhang, Jhang, Pakistan
}

Correspondence should be addressed to Jafar Tavoosi; j.tavoosi@ilam.ac.ir and Saleh Mobayen; mobayens@yuntech.edu.tw

Received 28 June 2021; Revised 28 July 2021; Accepted 19 August 2021; Published 7 September 2021

Academic Editor: Jesus Vega

Copyright ( $\odot 2021$ Jafar Tavoosi et al. This is an open access article distributed under the Creative Commons Attribution License, which permits unrestricted use, distribution, and reproduction in any medium, provided the original work is properly cited.

In industrial steam systems, the process requires a specific pressure, and the maximum permissible operating pressure is different. If the inlet steam pressure to the steam consuming equipment exceeds the operating pressure, it may cause hazards. Therefore, the more precise control of the boiler pressure is important. Since we are dealing with a nonlinear, time-varying, and multivariable system, the control method must be designed to handle this system well. Most of the methods proposed so far are either not physically feasible or the system has considered very simple. Therefore, in this paper, while modeling the boiler and its pressure relations more precisely, we will introduce a recurrent type- 2 fuzzy RBFN-based model reference adaptive control system with various uncertainties so that the uncertainty and inaccuracy of the model can be compensated. The experimental results prove the efficiency of the proposed method in boiler control.

\section{Introduction}

The chemical industries consume a great deal of energy to accomplish many tasks, including the process and supply of heat energy needed to heat materials. Current energy sources in the world mainly include fossil fuels, water and wind, and solar and nuclear energy. Of these, fossil fuels are used more often than any other source to supply energy carriers. Usually, in the chemical industry, carriers are electricity and steam. The best way to use fossil fuel energy to generate electricity and steam is to burn it [1]. In the burning process, the internal energy is released as heat and transferred to the environment in the form of radiation and displacement. Boilers and gas turbines are equipment commonly used to generate steam and electricity by burning fossil fuels. The boiler is a nonlinear, multivariable, and time-varying system and therefore requires an advanced control method [2].
Various articles have been presented in the field of thermal management in industries [3-5]. Here are some of the latest ones. In [6], a method for measuring the water level of the boiler is provided. The control of the boiler drum level is investigated using a feedback method. A laboratory-scale steam boiler, a small version of the power plant boiler, is intended for research in this article. In [7], fuzzy control was used to control the boiler with sugarcane fuel. This article uses a tool to find the dynamics of plant and set up control loops. In [8], a fractional order PID controller is introduced to study the temperature and pressure effects. In this paper, the AT89C55-based boiler water supply control system is designed and a simple fractional order PID algorithm is suggested. Also, the control of boiler water temperature and pressure simulation analysis has been performed. In [9], the integration of two-state model predictive control algorithms (OPM, optimal control predictive model) and generalized- 
predictive control (GPC) to fit a self-control system to an advanced cylindrical boiler dynamics $\mathrm{O}$ is performed with nonminimal phase behavior.

Not much has been done about controlling the boiler reference model. Perhaps, this is due to the over-dependence of the method on the boiler mathematical model, and since the boiler system is highly nonlinear and time varying, modelbased control may not be applicable in practice. However, some of the work done in this area is discussed below. In [10], the controller design for a boiler system with uncertainty is presented. To achieve this goal, a controller is designed for the class of multi-input nonlinear systems that correspond to the boiler equations. Computational intelligence is increasingly being developed [11-15]. Neural network-based control systems [16-20], fuzzy system [21-23], and fuzzy neural networks [24-27] have shown good performance. The following discusses some methods of boiler control based on computational intelligence. In [28], neural network-based predictive control is used to control carbon dioxide emissions from a large industrial boiler. In [29], fuzzy control is used to regulate the oxygen content of a boiler in the sugarcane industry. It has been shown in this study that fuzzy control leads to a decrease in metadata and a simultaneous increase in system response. In [30], a hierarchical fuzzy system is used to control a power boiler. It is acknowledged in the article that the proportional-integrator controller alone is not well responsive due to its complex, nonlinear, and inertial boiler nature and should therefore use with an intelligent control system. Very rare work on the neural network composition and model reference control method for boiler control is presented, which will be discussed below. In [31], the neural controller is presented. It is hard to obtain proper proficiency for the combustion controller of chain boilers due to the high latency, different quality of coal and load. A neural identification approach for time delay in nonlinear system is presented. Deficiencies [32] can be attributed to the lack of parametric and nonparametric uncertainty. In [33], a neural network radial basis function is used to model the boiler dyamics, and then, it is used in a model reference method. In other words, in this paper, the model reference block is a neural network model and it is a PID controller. The major drawback of [33] is the use of PID in the boiler system because the boiler is a nonlinear and delayed system and the PID does not provide a good response. Multivariable control means simultaneous control of parameters by considering their interaction $[34,35]$. Due to the complexity of simultaneously controlling all parameters, unfortunately, in most articles, the parameters are controlled individually. Certainly, in a multivariable system such as a boiler, it is better to control all the parameters at the same time, considering their effect on each other. So, the innovations of this article are

(1) Accurate modeling of an industrial and practical boiler with all the limitations and uncertainties

(2) Present recurrent type-2 fuzzy neural network in model reference control of a boiler for the first time

(3) Consider the interaction of variables with each other and use multivariable control
In this paper, first, the nonlinear equations of a real boiler are extracted, and then, the linear state space model is calculated to obtain the reference model. In the following, the multivariable control system of the model reference based on the proposed recurrent type-2 fuzzy RBFN is presented, and then, the rules of updating the neural network are presented. Finally, by simulating a real power plant boiler, the proposed control method is evaluated.

\section{Mathematical Modeling of the Boiler System}

The nonlinear dynamic of boiler-turbine-generator provided by Bell and Astrom is used. Since this model is extracted from the data of a real system in Sweden, this model has been used in most research projects since 1987 [36]. As shown in Figure 1, $y_{1}$ is the steam pressure of the drum in kilograms per square centimeter $\left(\mathrm{kg} / \mathrm{cm}^{2}\right), y_{2}$ is the electrical output power in megawatts (MW), and $y_{3}$ is the drum water level in terms of meter $(\mathrm{m})$ The output variables of this system are multivariable. Also, the flow rate of the input fuel, the $u_{2}$ flow rate of the boiler output steam and the turbine inlet, and the $u_{3}$ flow rate of the water entering the boiler are the input variables. So, we have a multivariable system of three inputs and three outputs. Table 1 shows the parametric values of the system.

The state-of-the-art form of this multivariable system with a power of $160 \mathrm{MW}$ is as follows:

$$
\begin{aligned}
& \dot{x}_{1}=-0.0018 u_{2} x_{1}^{9 / 8}+0.9 u_{1}-0.15 u_{3}, \\
& \dot{x}_{2}=\frac{\left(0.73 u_{2}-0.16\right) x_{1}^{9 / 8}-x_{2}}{10}, \\
& \dot{x}_{3}=\frac{\left[141 u_{3}-\left(1.1 u_{2}-0.19\right) x_{1}\right]}{85}, \\
& y_{1}=x_{1}, \\
& y_{2}=x_{2}, \\
& y_{3}=0.05\left(0.13073 x_{3}+100 a_{c s}+q_{e}-67.975\right) .
\end{aligned}
$$

In the above system, $x_{1}$ is the steam pressure of the drum, $x_{2}$ is the power output, $x_{3}$ is the steam density inside the drum in terms of $\mathrm{kg} / \mathrm{m}^{3}, u_{1}$ is the amount of flow input fuel, $u_{2}$ is the amount of steam discharge applied to the turbine from the boiler, and $u_{3}$ is the flow of water entering the drum. Also, $a_{\mathrm{cs}}$ and $q_{e}$ are the rate of steam quality and evaporation $(\mathrm{kg} / \mathrm{s})$ and are calculated by the following equations. It should be noted that $y_{3}$ is the water level change of the drum:

$$
\begin{aligned}
a_{\mathrm{cs}} & =\frac{\left(-25.6+0.8 x_{1}\right)\left(1-0.001538 x_{3}\right)}{\left(1.0394-0.0012340 x_{1}\right) x_{3}}, \\
q_{e} & =x_{1}\left(0.854 u_{4}-0.147\right)+45.59 u_{1}-2.514 u_{3}-2.096 .
\end{aligned}
$$

To design a model reference control system, one must extract a linear model of the system that is easily controllable 


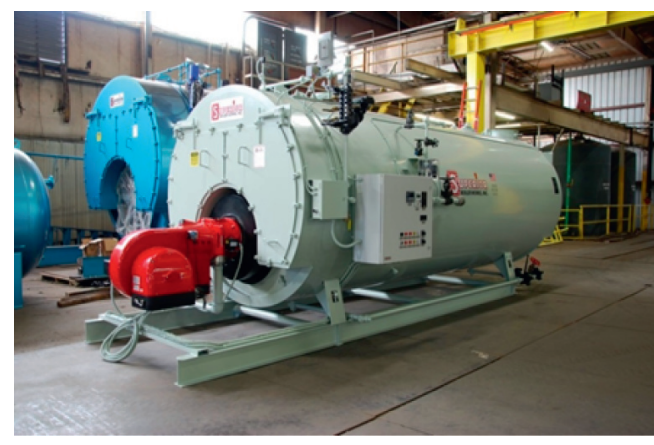

FIgURE 1: A power plant boiler in Iran.

TABLE 1: System parameter values.

\begin{tabular}{lc}
\hline Nominal power & $160 \mathrm{MW}$ \\
\hline Rate of fuel flow & $14 \mathrm{~kg} / \mathrm{sec}$ \\
Rate of vapor flow & $140 \mathrm{~kg} / \mathrm{sec}$ \\
Inlet temperature & $3^{\circ} \mathrm{C}$ \\
Steam pressure & $140 \mathrm{~kg} / \mathrm{cm}^{2}$ \\
Steam mass & $2000 \mathrm{~kg}$ \\
Steam temperature & $535^{\circ} \mathrm{C}$ \\
Drama size & $40 \mathrm{~m}^{3}$ \\
Water mass & $40000 \mathrm{~kg}$ \\
\hline
\end{tabular}

and design a controller for it. In this case, since the behavior of the linear model is similar to the main nonlinear model, the main nonlinear model can also be controlled by careful control of the linear model.

\section{Linearization of the Nonlinear Model of the Boiler}

Boiler-turbine-generator system is one of the nonlinear and multivariable models. In this dissertation, the nonlinear model is linearized as

$$
\begin{aligned}
& y^{0}=\left(y_{1}^{0}, y_{2}^{0}, y_{3}^{0}\right), \\
& x^{0}=\left(x_{1}^{0}, x_{2}^{0}, x_{3}^{0}\right), \\
& u^{0}=\left(u_{1}^{0}, u_{2}^{0}, u_{3}^{0}\right) .
\end{aligned}
$$

The general form of nonlinear systems is $\dot{x}=f(x, u)$, which can be written as

$$
\begin{aligned}
\frac{\mathrm{d} x_{1}}{\mathrm{~d} t} & =f_{1}\left(x_{1}, x_{2}, \ldots, x_{n}, u_{1}, u_{2}, \ldots, u_{m}\right), \\
\frac{\mathrm{d} x_{2}}{\mathrm{~d} t} & =f_{2}\left(x_{1}, x_{2}, \ldots, x_{n}, u_{1}, u_{2}, \ldots, u_{m}\right), \\
& \vdots \\
\frac{\mathrm{d} x_{n}}{\mathrm{~d} t} & =f_{n}\left(x_{1}, x_{2}, \ldots, x_{n}, u_{1}, u_{2}, \ldots, u_{m}\right) .
\end{aligned}
$$

Assume that $u^{0}=\left[\begin{array}{llll}u_{1}^{0} & u_{2}^{0} & \ldots & u_{m}^{0}\end{array}\right]^{T}$ is entered as a constant input to the system $\dot{x}=f(x, u)$ and balance it to $x^{0}=\left[\begin{array}{llll}x_{1}^{0} & x_{2}^{0} & \ldots & x_{n}^{0}\end{array}\right]^{T}$. So,

$$
\begin{aligned}
& x=x^{0}+\Delta x, \\
& u=u^{0}+\Delta u .
\end{aligned}
$$

Using the Taylor extension, we will have

$$
\begin{aligned}
\frac{\mathrm{d} x}{\mathrm{~d} t}= & f\left(x^{0}+\Delta x, u^{0}+\Delta u\right) \\
= & f\left(x^{0}, u^{0}\right)+\frac{\mathrm{d} f}{\mathrm{~d} x}\left(x^{0}, u^{0}\right) \Delta x+\frac{\mathrm{d} f}{\mathrm{~d} u}\left(x^{0}, u^{0}\right) \Delta u \\
& +\frac{1}{2} \frac{\partial^{2} f}{\partial x^{2}}\left(x^{0}, u^{0}\right)(\Delta x)^{2} \\
& +\frac{\partial^{2} f}{\partial x \partial u}\left(x^{0}, u^{0}\right)(\Delta x)(\Delta u)+\frac{1}{2} \frac{\partial^{2} f}{\partial u^{2}}\left(x^{0}, u^{0}\right)(\Delta u)^{2} .
\end{aligned}
$$

So, regardless of the nonlinear terms, we will have

$$
\frac{\mathrm{d} x}{\mathrm{~d} t}=f\left(x^{0}, u^{0}\right)+\frac{\mathrm{d} f}{\mathrm{~d} x}\left(x^{0}, u^{0}\right) \Delta x+\frac{\mathrm{d} f}{\mathrm{~d} u}\left(x^{0}, u^{0}\right) \Delta u,
$$

where

$$
\begin{aligned}
\frac{\mathrm{d} f}{\mathrm{~d} x}\left(x^{0}, u^{0}\right) & =\left[\begin{array}{ccc}
\frac{\mathrm{d} f_{1}}{\mathrm{~d} x_{1}} & \cdots & \frac{\mathrm{d} f_{1}}{\mathrm{~d} x_{n}} \\
\vdots & \ddots & \vdots \\
\frac{\mathrm{d} f_{n}}{\mathrm{~d} x_{1}} & \cdots & \frac{\mathrm{d} f_{n}}{\mathrm{~d} x_{n}}
\end{array}\right]_{\left(x^{0}, u^{0}\right)}, \\
\frac{\mathrm{d} f}{\mathrm{~d} u}\left(x^{0}, u^{0}\right) & =\left[\begin{array}{ccc}
\frac{\mathrm{d} f_{1}}{\mathrm{~d} u_{1}} & \cdots & \frac{\mathrm{d} f_{1}}{\mathrm{~d} u_{n}} \\
\vdots & \ddots & \vdots \\
\frac{\mathrm{d} f_{n}}{\mathrm{~d} u_{1}} & \cdots & \frac{\mathrm{d} f_{n}}{\mathrm{~d} u_{n}}
\end{array}\right]_{\left(x^{0}, u^{0}\right)} .
\end{aligned}
$$

We also have

$$
\frac{\mathrm{d} x}{\mathrm{~d} t}=\frac{\mathrm{d} x^{0}}{\mathrm{~d} t}+\frac{\mathrm{d}(\Delta x)}{\mathrm{d} t}=\frac{\mathrm{d}(\Delta x)}{\mathrm{d} t}
$$

because $x^{0}$ is a fixed number. Assume

$$
\begin{aligned}
& A=\frac{\mathrm{d} f}{\mathrm{~d} x}\left(x^{0}, u^{0}\right), \\
& B=\frac{\mathrm{d} f}{\mathrm{~d} u}\left(x^{0}, u^{0}\right) .
\end{aligned}
$$

By ignoring higher-order sentences to achieve linear approximation and writing in the form of state space, 


$$
\frac{\mathrm{d}(\Delta x)}{\mathrm{d} t}=A \Delta x+B \Delta u
$$

Similarly, the output of the nonlinear system model is as follows:

$$
\begin{aligned}
y_{1} & =h_{1}\left(x_{1}, x_{2}, \ldots, x_{n}, u_{1}, u_{2}, \ldots, u_{m}\right), \\
y_{2} & =h_{2}\left(x_{1}, x_{2}, \ldots, x_{n}, u_{1}, u_{2}, \ldots, u_{m}\right), \\
& \vdots \\
y_{n} & =h_{n}\left(x_{1}, x_{2}, \ldots, x_{n}, u_{1}, u_{2}, \ldots, u_{m}\right) .
\end{aligned}
$$

In other words,

$$
y=h(x, u)
$$

The expansion of the Taylor series is again used as

$$
y=y^{0}+\Delta y
$$

So, we will have

$$
\begin{aligned}
h(x, u) & =h\left(x^{0}, u^{0}\right)+\frac{\mathrm{d} h}{\mathrm{~d} x}\left(x^{0}, u^{0}\right) \Delta x+\frac{\mathrm{d} h}{\mathrm{~d} u}\left(x^{0}, u^{0}\right) \Delta u, \\
y^{0} & =h\left(x^{0}, u^{0}\right) .
\end{aligned}
$$

So,

$$
\begin{aligned}
y-y^{0} & =\frac{\mathrm{d} h}{\mathrm{~d} x}\left(x^{0}, u^{0}\right) \Delta x+\frac{\mathrm{d} h}{\mathrm{~d} u}\left(x^{0}, u^{0}\right) \Delta u, \\
\Delta y & =\bar{y}=C \bar{x}+D \bar{u},
\end{aligned}
$$

where

$$
\begin{aligned}
& C=\frac{\mathrm{d} h}{\mathrm{~d} x}\left(x^{0}, u^{0}\right), \\
& D=\frac{\mathrm{d} h}{\mathrm{~d} u}\left(x^{0}, u^{0}\right), \\
& \bar{y}=\Delta y=\left(y-y^{0}\right), \\
& \bar{x}=\Delta x=\left(x-x^{0}\right), \\
& \bar{u}=\Delta u=\left(u-u^{0}\right) .
\end{aligned}
$$

Now, we can write the general vector symbol of the nonlinear system and the space model mode of the linear system as

$$
\begin{aligned}
& \dot{x}=f(x, u), \\
& y=h(x, u),
\end{aligned}
$$

and linear form is

$$
\begin{aligned}
& \dot{x}=A x+B u, \\
& y=C x+D u,
\end{aligned}
$$

where the linear matrix elements are as follows:

$$
\begin{aligned}
& A_{i j}=\frac{\partial f_{i}}{\partial x_{j}}\left(x^{0}, u^{0}\right), \\
& B_{i j}=\frac{\partial f_{i}}{\partial u_{j}}\left(x^{0}, u^{0}\right), \\
& C_{i j}=\frac{\partial h_{i}}{\partial x_{j}}\left(x^{0}, u^{0}\right), \\
& D_{i j}=\frac{\partial h_{i}}{\partial u_{j}}\left(x^{0}, u^{0}\right) .
\end{aligned}
$$

To implement the above linearization theory on the boiler system, the following is done:

$$
\begin{aligned}
A_{11} & =\frac{\partial f_{1}}{\partial x_{1}}\left(x^{0}, u^{0}\right)=\frac{\partial}{\partial x_{1}}\left(-0.0018 u_{2} x_{1}^{9 / 8}+0.9 u_{1}-0.15 u_{3}\right)_{\left(x^{0}, u^{0}\right)} \\
& =-0.0018 \frac{9}{8} u_{2}^{0}\left(x_{1}^{0}\right)^{1 / 8}=-0.00203 u_{2}^{0}\left(x_{1}^{0}\right)^{1 / 8}, \\
A_{12} & =\frac{\partial f_{1}}{\partial x_{2}}\left(x^{0}, u^{0}\right)=\frac{\partial}{\partial x_{2}}\left(-0.0018 u_{2} x_{1}^{9 / 8}+0.9 u_{1}-0.15 u_{3}\right)_{\left(x^{0}, u^{0}\right)}=0, \\
A_{13} & =\frac{\partial f_{1}}{\partial x_{3}}\left(x^{0}, u^{0}\right)=\frac{\partial}{\partial x_{3}}\left(-0.0018 u_{2} x_{1}^{9 / 8}+0.9 u_{1}-0.15 u_{3}\right)_{\left(x^{0}, u^{0}\right)}=0, \\
A_{21} & =\frac{\partial f_{2}}{\partial x_{1}}\left(x^{0}, u^{0}\right)=\frac{\partial}{\partial x_{1}}\left(\frac{\left(0.73 u_{2}-0.16\right) x_{1}^{9 / 8}-x_{2}}{10}\right)_{\left(x^{0}, u^{0}\right)}
\end{aligned}
$$

$$
\begin{aligned}
= & \frac{9}{8}\left(\left(0.73 u_{2}-0.16\right) x_{1}^{9 / 8}\right)_{\left(x^{0}, u^{0}\right)}=\left(0.08212 u_{2}^{0}-0.018\right)\left(x_{1}^{0}\right)^{\frac{1}{8}} \\
A_{22}= & \frac{\partial f_{2}}{\partial x_{2}}\left(x^{0}, u^{0}\right)=\frac{\partial}{\partial x_{2}}\left(\frac{\left(0.73 u_{2}-0.16\right) x_{1}^{9 / 8}-x_{2}}{10}\right)_{\left(x^{0}, u^{0}\right)}=-0.1, \\
A_{23}= & \frac{\partial f_{2}}{\partial x_{3}}\left(x^{0}, u^{0}\right)=\frac{\partial}{\partial x_{3}}\left(\frac{\left(0.73 u_{2}-0.16\right) x_{1}^{9 / 8}-x_{2}}{10}\right)_{\left(x^{0}, u^{0}\right)}=0, \\
A_{31}= & \frac{\partial f_{3}}{\partial x_{1}}\left(x^{0}, u^{0}\right)=\frac{\partial}{\partial x_{1}}\left(\frac{\left[141 u_{3}-\left(1.1 u_{2}-0.19\right) x_{1}\right]}{85}\right)_{\left(x^{0}, u^{0}\right)} \\
= & \frac{\left(0.19-1.1 u_{2}^{0}\right)}{85}
\end{aligned}
$$$$
A_{32}=\frac{\partial f_{3}}{\partial x_{2}}\left(x^{0}, u^{0}\right)=\frac{\partial}{\partial x_{2}}\left(\frac{\left[141 u_{3}-\left(1.1 u_{2}-0.19\right) x_{1}\right]}{85}\right)_{\left(x^{0}, u^{0}\right)}=0,
$$$$
A_{33}=\frac{\partial f_{3}}{\partial x_{1}}\left(x^{0}, u^{0}\right)=\frac{\partial}{\partial x_{1}}\left(\frac{\left[141 u_{3}-\left(1.1 u_{2}-0.19\right) x_{1}\right]}{85}\right)_{\left(x^{0}, u^{0}\right)}=0 .
$$

Matrix B drives are also calculated as follows: 


$$
\begin{aligned}
& B_{11}=\frac{\partial f_{1}}{\partial u_{1}}\left(x^{0}, u^{0}\right)=\frac{\partial}{\partial u_{1}}\left(-0.0018 u_{2} x_{1}^{9 / 8}+0.9 u_{1}-0.15 u_{3}\right)_{\left(x^{0}, u^{0}\right)}=0.9 \\
& B_{12}=\frac{\partial f_{1}}{\partial u_{2}}\left(x^{0}, u^{0}\right)=\frac{\partial}{\partial u_{2}}\left(-0.0018 u_{2} x_{1}^{9 / 8}+0.9 u_{1}-0.15 u_{3}\right)_{\left(x^{0}, u^{0}\right)}=-0.0018\left(x_{1}^{0}\right)^{9 / 8} \text {, } \\
& B_{13}=\frac{\partial f_{1}}{\partial u_{3}}\left(x^{0}, u^{0}\right)=\frac{\partial}{\partial u_{3}}\left(-0.0018 u_{2} x_{1}^{9 / 8}+0.9 u_{1}-0.15 u_{3}\right)_{\left(x^{0}, u^{0}\right)}=-0.15 \text {, } \\
& B_{21}=\frac{\partial f_{2}}{\partial u_{1}}\left(x^{0}, u^{0}\right)=\frac{\partial}{\partial u_{1}}\left(\frac{\left(0.73 u_{2}-0.16\right) x_{1}^{9 / 8}-x_{2}}{10}\right)_{\left(x^{0}, u^{0}\right)}=0 \\
& B_{22}=\frac{\partial f_{2}}{\partial u_{2}}\left(x^{0}, u^{0}\right)=\frac{\partial}{\partial u_{2}}\left(\frac{\left(0.73 u_{2}-0.16\right) x_{1}^{9 / 8}-x_{2}}{10}\right)_{\left(x^{0}, u^{0}\right)}=0.073\left(x_{1}^{0}\right)^{9 / 8}, \\
& B_{23}=\frac{\partial f_{2}}{\partial u_{3}}\left(x^{0}, u^{0}\right)=\frac{\partial}{\partial u_{3}}\left(\frac{\left(0.73 u_{2}-0.16\right) x_{1}^{9 / 8}-x_{2}}{10}\right)_{\left(x^{0}, u^{0}\right)}=0 \\
& B_{31}=\frac{\partial f_{3}}{\partial u_{1}}\left(x^{0}, u^{0}\right)=\frac{\partial}{\partial u_{1}}\left(\frac{\left[141 u_{3}-\left(1.1 u_{2}-0.19\right) x_{1}\right]}{85}\right)_{\left(x^{0}, u^{0}\right)}=0 \\
& B_{32}=\frac{\partial f_{3}}{\partial u_{2}}\left(x^{0}, u^{0}\right)=\frac{\partial}{\partial u_{2}}\left(\frac{\left[141 u_{3}-\left(1.1 u_{2}-0.19\right) x_{1}\right]}{85}\right)_{\left(x^{0}, u^{0}\right)}=-\frac{1.1}{85} x_{1}^{0}, \\
& B_{33}=\frac{\partial f_{3}}{\partial u_{3}}\left(x^{0}, u^{0}\right)=\frac{\partial}{\partial u_{1}}\left(\frac{\left[141 u_{3}-\left(1.1 u_{2}-0.19\right) x_{1}\right]}{85}\right)_{\left(x^{0}, u^{0}\right)}=\frac{141}{85}
\end{aligned}
$$

The following are also used to determine the drives of matrices $C$ and $D$ :

$$
\begin{aligned}
& C_{11}= \frac{\partial h_{1}}{\partial x_{1}}\left(x^{0}, u^{0}\right)=\frac{\partial}{\partial x_{1}}\left(x_{1}\right)_{\left(x^{0}, u^{0}\right)}=1, \\
& C_{12}= \frac{\partial h_{1}}{\partial x_{2}}\left(x^{0}, u^{0}\right)=\frac{\partial}{\partial x_{2}}\left(x_{1}\right)_{\left(x^{0}, u^{0}\right)}=0, \\
& C_{13}= \frac{\partial h_{1}}{\partial x_{3}}\left(x^{0}, u^{0}\right)=\frac{\partial}{\partial x_{3}}\left(x_{1}\right)_{\left(x^{0}, u^{0}\right)}=0, \\
& C_{21}= \frac{\partial h_{2}}{\partial x_{1}}\left(x^{0}, u^{0}\right)=\frac{\partial}{\partial x_{1}}\left(x_{1}\right)_{\left(x^{0}, u^{0}\right)}=0, \\
& C_{22}= \frac{\partial h_{2}}{\partial x_{2}}\left(x^{0}, u^{0}\right)=\frac{\partial}{\partial x_{2}}\left(x_{1}\right)_{\left(x^{0}, u^{0}\right)}=1, \\
& C_{23}= \frac{\partial h_{2}}{\partial x_{3}}\left(x^{0}, u^{0}\right)=\frac{\partial}{\partial x_{3}}\left(x_{1}\right)_{\left(x^{0}, u^{0}\right)}=0, \\
& C_{31}= \frac{\partial h_{3}}{\partial x_{1}}\left(x^{0}, u^{0}\right)=\frac{\partial}{\partial x_{1}}\left(0.05\left(0.13073 x_{3}+100 a_{c s}+q_{e}-67.975\right)\right)\left(x^{0}, u^{0}\right) \\
&
\end{aligned}
$$


where

$$
\begin{aligned}
\frac{\partial a_{\mathrm{cs}}}{\partial x_{1}}\left(x^{0}, u^{0}\right) & =\frac{\left[x_{3}^{0}\left(1.0394-0.00123404 x_{1}^{0}\right)\left(0.8-0.00123404 x_{3}^{0}\right)-\right]}{\left(x_{3}^{0}\left(1.0394-0.00123404 x_{1}^{0}\right)\right)^{2}}-\frac{\left(1-0.001538 x_{3}^{0}\right)\left(0.8 x_{1}^{0}-25.6\right)\left(-0.00123404 x_{3}^{0}\right)}{\left(x_{3}^{0}\left(1.0394-0.00123404 x_{1}^{0}\right)\right)^{2}} \\
\frac{\partial q_{e}}{\partial x_{1}}\left(x^{0}, u^{0}\right) & =0.854 u_{2}^{0}-0.147 \\
C_{32} & =\frac{\partial h_{3}}{\partial x_{2}}\left(x^{0}, u^{0}\right)=\frac{\partial}{\partial x_{2}}\left(0.05\left(0.13073 x_{3}+100 a_{c s}+q_{e}-67.975\right)\right)_{\left(x^{0}, u^{0}\right)}=0 \\
C_{33} & =\frac{\partial h_{3}}{\partial x_{3}}\left(x^{0}, u^{0}\right)=\frac{\partial}{\partial x_{3}}\left(0.05\left(0.13073 x_{3}+100 a_{c s}+q_{e}-67.975\right)\right)_{\left(x^{0}, u^{0}\right)}=0.0065365+5 \frac{\partial a_{\mathrm{cs}}}{\partial x_{3}}
\end{aligned}
$$

where

$$
\begin{aligned}
& \frac{\partial a_{\mathrm{cs}}}{\partial x_{3}}= \frac{\left[x_{3}^{0}\left(1.0394-0.00123404 x_{1}^{0}\right)\left(0.00123404 x_{1}^{0}+0.039372\right)\right]}{\left(x_{3}^{0}\left(1.0394-0.00123404 x_{1}^{0}\right)\right)^{2}}\left(1-0.001538 \mathrm{x}_{3}^{0}\right)\left(0.8 \mathrm{x}_{1}^{0}-25.6\right)\left(-0.00123404 \mathrm{x}_{1}^{0}\right) \\
& \cdot\left(x_{3}^{0}\left(1.0394-0.00123404 x_{1}^{0}\right)\right)^{2} \\
& D_{11}= \frac{\partial h_{1}}{\partial u_{1}}\left(x^{0}, u^{0}\right)=\frac{\partial}{\partial u_{1}}\left(x_{1}\right)_{\left(x^{0}, u^{0}\right)}=0 \\
& D_{12}= \frac{\partial h_{1}}{\partial u_{2}}\left(x^{0}, u^{0}\right)=\frac{\partial}{\partial u_{2}}\left(x_{1}\right)_{\left(x^{0}, u^{0}\right)}=0 \\
& D_{13}= \frac{\partial h_{1}}{\partial u_{3}}\left(x^{0}, u^{0}\right)=\frac{\partial}{\partial u_{3}}\left(x_{1}\right)_{\left(x^{0}, u^{0}\right)}=0 \\
& D_{21}= \frac{\partial h_{2}}{\partial u_{1}}\left(x^{0}, u^{0}\right)=\frac{\partial}{\partial u_{1}}\left(x_{2}\right)_{\left(x^{0}, u^{0}\right)}=0 \\
& D_{22}= \frac{\partial h_{2}}{\partial u_{2}}\left(x^{0}, u^{0}\right)=\frac{\partial}{\partial u_{2}}\left(x_{2}\right)_{\left(x^{0}, u^{0}\right)}=0 \\
& D_{23}= \frac{\partial h_{2}}{\partial u_{3}}\left(x^{0}, u^{0}\right)=\frac{\partial}{\partial u_{3}}\left(x_{2}\right)_{\left(x^{0}, u^{0}\right)}=0 \\
& D_{31}= \frac{\partial h_{3}}{\partial u_{1}}\left(x^{0}, u^{0}\right) \\
&= \frac{\partial}{\partial u_{1}}\left(0.05\left(0.13073 x_{3}+100 a_{\mathrm{cs}}+q_{e}-67.975\right)\right)_{\left(x^{0}, u^{0}\right)}=0.2279 \\
& D_{32}= \frac{\partial h_{3}}{\partial u_{2}}\left(x^{0}, u^{0}\right) \\
&= \frac{\partial}{\partial u_{1}}\left(0.05\left(0.13073 x_{3}+100 a_{\mathrm{cs}}+q_{e}-67.975\right)\right)_{\left(x^{0}, u^{0}\right)}=0.00427 x_{1}^{0} \\
& D_{33}= \frac{\partial h_{3}}{\partial u_{3}}\left(x^{0}, u^{0}\right) \\
&= \frac{\partial}{\partial u_{3}}\left(0.05\left(0.13073 x_{3}+100 a_{\mathrm{cs}}+q_{e}-67.975\right)\right)_{\left(x^{0}, u^{0}\right)}=-0.014 \\
&
\end{aligned}
$$


Therefore, four matrices $A, B, C$, and $D$ are obtained as follows.

$$
A=\left[\begin{array}{ccc}
-0.00203 u_{2}^{0}\left(x_{1}^{0}\right)^{1 / 8} & 0 & 0 \\
\left(0.08212 u_{2}^{0}-0.018\right)\left(x_{1}^{0}\right)^{1 / 8} & -0.1 & 0 \\
\frac{\left(0.19-1.1 u_{2}^{0}\right)}{85} & 0 & 0
\end{array}\right],
$$$$
B=\left[\begin{array}{ccc}
0.9 & -0.0018\left(x_{1}^{0}\right)^{9 / 8} & -0.15 \\
0 & 0.073\left(x_{1}^{0}\right)^{9 / 8} & 0 \\
0 & -\frac{1.1}{85} x_{1}^{0} & \frac{141}{85}
\end{array}\right] \text {, }
$$

$$
C=\left[\begin{array}{ccc}
1 & 0 & 0 \\
0 & 1 & 0 \\
5 \frac{\partial a_{\mathrm{cs}}}{\partial x_{1}}+0.05 \frac{\partial q_{e}}{\partial x_{1}} & 0 & 0.0065365+5 \frac{\partial a_{\mathrm{cs}}}{\partial x_{3}}
\end{array}\right],
$$

$$
D=\left[\begin{array}{ccc}
0 & 0 & 0 \\
0 & 0 & 0 \\
0.2279 & 0.00427 x_{1}^{0} & -0.014
\end{array}\right] \text {. }
$$
boiler:

Therefore, a linear state space form can be written for the

$$
\begin{aligned}
& \dot{x}=A x+B u, \\
& y=C x+D u .
\end{aligned}
$$

The variables $y, x$, and $u$ are the output, mode, and input of the boiler system at the operating points, respectively.

\section{Neural Model Reference Adaptive Control}

Here, the controller structure is described (Figure 2). In the previous section, the linear model of the boiler was obtained, and it is used in the model reference block.

In Figure 2, the control system calculates the difference between the boiler output and the reference model. This error signal is then used to train the recurrent type- 2 fuzzy radial basis function neural network (RT2FRBFN) [37]. We train the neural network so that the error is less and less, and as a result, the boiler output follows the output of the reference model. In this case, the system is controlled comparatively. Lyapunov's sustainability rule design approach is one of the most important methods of adaptive control [38-40]. This method tries to find the Lyapunov function and a matching mechanism so that the error between the system and the model is zero. This method also ensures the stability of the control parameters. The model reference is usually defined as a first-order system as follows:

$$
\frac{\mathrm{d} y_{m}}{\mathrm{~d} t}=-a_{m} y_{m}+b_{m} u_{c}
$$

where $y_{m}$ are the output of the model reference and $u_{c}$ are the input of the reference model. Suppose the system to be controlled is as follows:

$$
\frac{\mathrm{d} y}{\mathrm{~d} t}=-a y+b u
$$

where $y$ is the output of the system and $u$ is its input. The control signal with the adjustable parameters $\theta_{1}$ and $\theta_{2}$ is selected as follows:

$$
u=\theta_{1} u_{c}-\theta_{2} y
$$

The error is calculated as follows:

$$
e=y-y_{m}
$$

Since the above error must be zero, the error changes must be negative. So,

$$
\begin{aligned}
\dot{e} & =\dot{y}-\dot{y}_{m} \\
& =-a y+b u-a_{m} y_{m}+b_{m} u_{c} \\
& =-a y+b u+a_{m} y_{m}-b_{m} u_{c} .
\end{aligned}
$$

So, we will have

$$
\begin{aligned}
\dot{e} & =-a y+b\left(\theta_{1} u_{c}-\theta_{2} y\right)+a_{m} y_{m}-b_{m} u_{c} \\
& =-a y-b \theta_{2} y+a_{m} y_{m}-\left(b \theta_{1}-b_{m}\right) u_{c} \\
& =-a_{m} e-\left(b \theta_{2}+a-a_{m}\right) y+\left(b \theta_{1}-b_{m}\right) u_{c} .
\end{aligned}
$$

To find the law of conformity, consider Lyapunov's function as follows:

$V\left(e, \theta_{1}, \theta_{2}\right)=\frac{1}{2}\left[e^{2}+\frac{1}{b \gamma}\left(b \theta_{2}+a-a_{m}\right)^{2}+\frac{1}{b \gamma}\left(b \theta_{1}-b_{m}\right)^{2}\right]$.

Based on the above Lyapunov function, we will have

$$
\begin{aligned}
\dot{V}= & e \frac{\mathrm{d} e}{\mathrm{~d} t}+\frac{1}{\gamma}\left(b \theta_{2}+a-a_{m}\right) \frac{\mathrm{d} \theta_{2}}{\mathrm{~d} t}+\frac{1}{\gamma}\left(b \theta_{1}-b_{m}\right) \frac{\mathrm{d} \theta_{1}}{\mathrm{~d} t} \\
= & -a_{m} e^{2}+\frac{1}{\gamma}\left(b \theta_{2}+a-a_{m}\right)\left(\frac{\mathrm{d} \theta_{2}}{\mathrm{~d} t}-\gamma y e\right) \\
& +\frac{1}{\gamma}\left(b \theta_{1}-b_{m}\right)\left(\frac{\mathrm{d} \theta_{1}}{\mathrm{~d} t}+\gamma u_{c} e\right) .
\end{aligned}
$$

In order for the above statement to be negative, we must have

$$
\begin{aligned}
& \frac{\mathrm{d} \theta_{1}}{\mathrm{~d} t}=-\gamma u_{c} e, \\
& \frac{\mathrm{d} \theta_{2}}{\mathrm{~d} t}=\gamma y e,
\end{aligned}
$$

where $\gamma$ is the learning rate. So, 


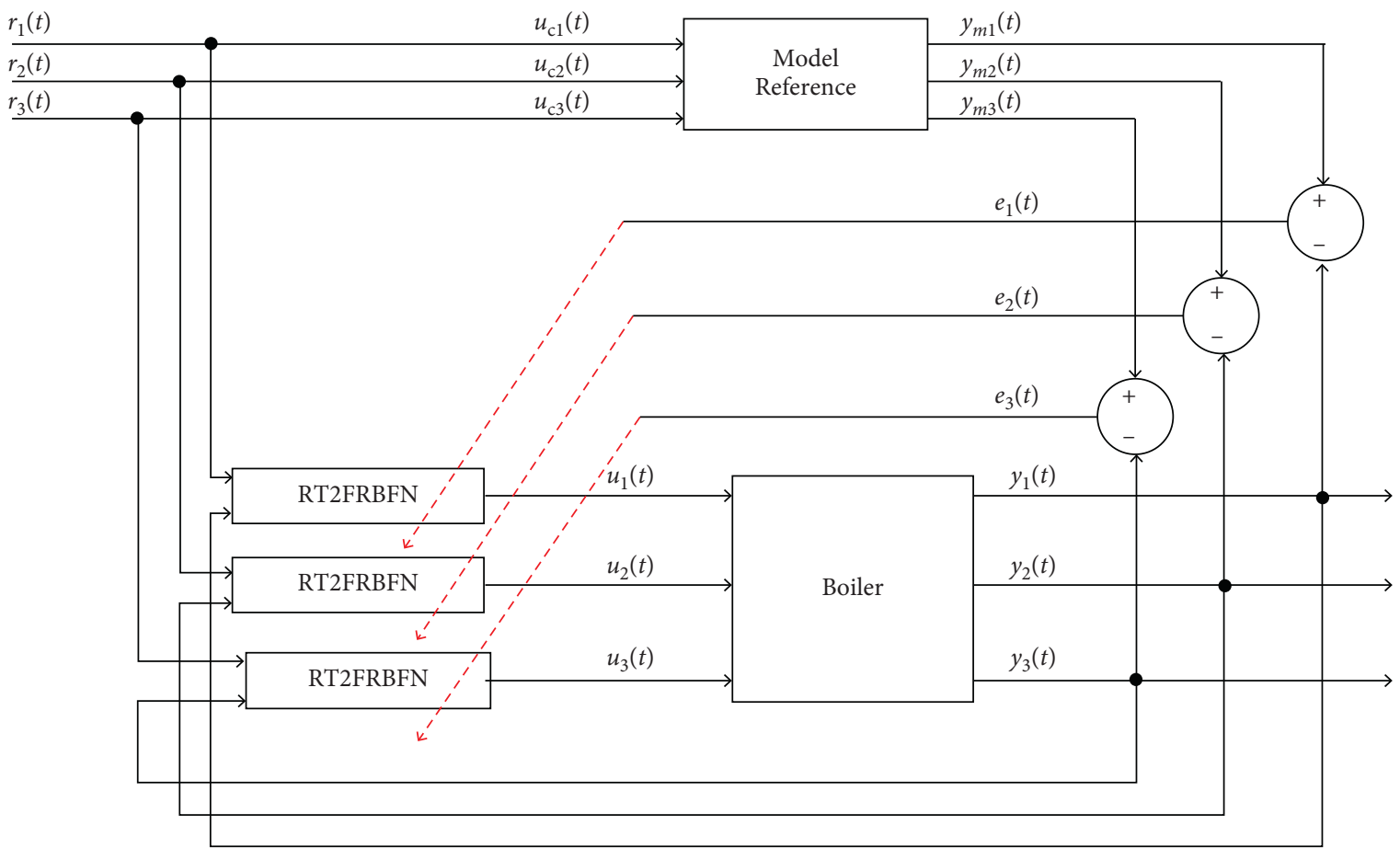

Figure 2: The suggested control structure.

$$
\dot{V}=-a_{m} e^{2}
$$

Therefore, the system will be stable and the change of parameters will be in order to reduce the error. The adaptation law for training the neural network uses equations (36) and (37).

\section{Experimental Results}

In this section, the performance of the control method of the proposed model reference for boiler control is examined. The industrial boiler is shown in Figure 3. To test the control system, assume that the boiler output steam pressure in the first 100 seconds is $150 \mathrm{~kg} / \mathrm{cm}^{2}$, from 200 seconds to 200 seconds, it is $300 \mathrm{~kg} / \mathrm{cm}^{2}$, and, finally, from 200 to 300 seconds per second, it is a value of $150 \mathrm{~kg} / \mathrm{cm}^{2}$.

Figure 4 illustrates the performance of the proposed neural controller.

In the following, the control of the electric power output as the second component of the boiler output that must be controlled is presented. Assume that, according to the above steam pressure control scenario, the output power is to be $50 \mathrm{~kW}$ in the first 100 seconds, $200 \mathrm{~kW}$ from 100 to 100 seconds, and, finally, $300 \mathrm{~kW}$ from 200 seconds to 300 seconds. Figure 5 illustrates the designed control performance in electric power control. Three perceptron neural networks have been used, and the number of hidden layer is 16,14 , and 17, respectively.

The following examines the control of the water level of the drum as the third component of the boiler output that should be controlled. Assume that, according to the boiler steam pressure control scenario, the water level of the drum is supposed to be $400 \mathrm{~cm}$ in the first 100 seconds, $200 \mathrm{~cm}$

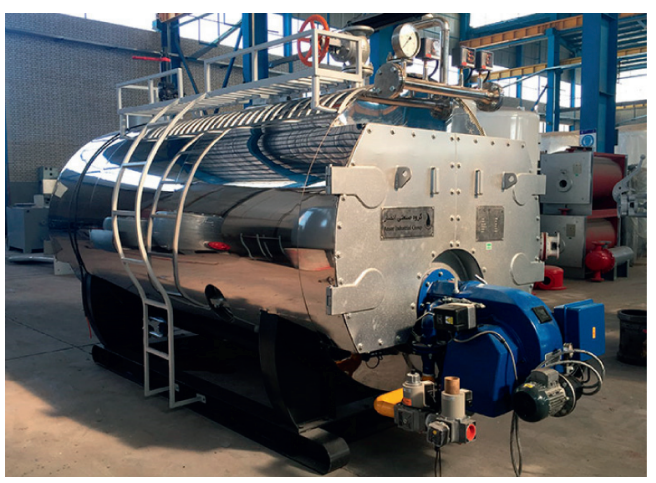

FIgURE 3: The industrial boiler.

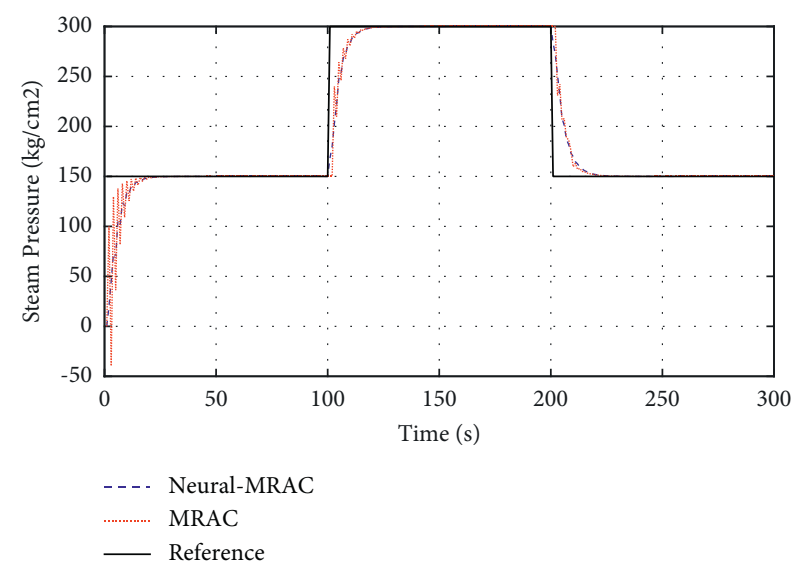

FIgURE 4: Control of the boiler steam pressure model reference. 


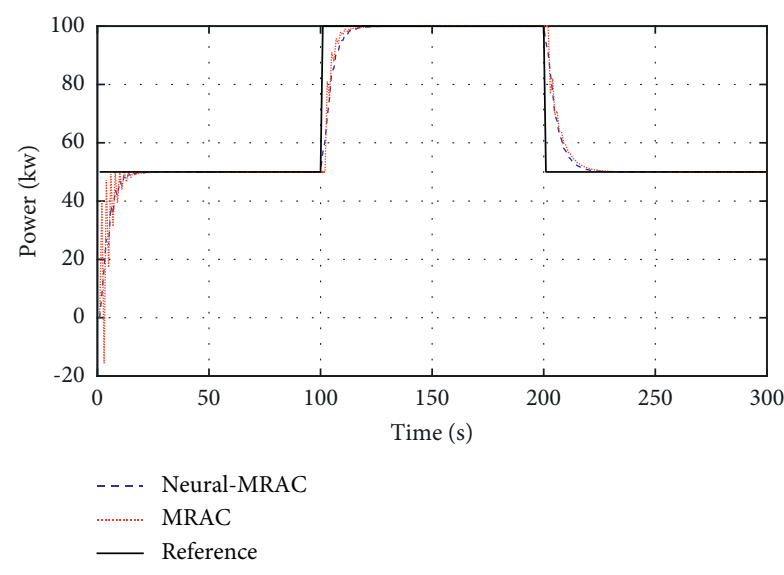

FIGURE 5: Control of the power reference model.

from 100 to $100 \mathrm{~s}$ in the second, and 300 to $400 \mathrm{~cm}$ in the second from 200 to 300 s. Figure 6 presents control performance in drum water level control.

The above results are related to an ideal boiler with constant coefficients. In other words, the coefficients and parameters of the boiler are considered unchanged with time; as in practice, some coefficients may change for various reasons, such as wear and tear over time, high heat, humidity, and environmental conditions. In this case, a proper controller must be robust to parametric variations. To check the performance of the proposed model reference control system in the presence of parametric changes, assume that the coefficients of each sentence in the equations of the boiler state space change by $\pm 10 \%$ and their nominal value randomly. In this case, the performance of the proposed reference control system model in boiler steam pressure control is shown in Figure 7.

Figure 8 shows the electrical power control outputs, despite the uncertain parameter.

In the following, the indefinite effect of the parameter in controlling the water level of the drum is examined. Figure 9 illustrates the control function of the reference water level model of the drum despite the uncertain parameter.

However, there is another uncertainty that stems from a lack of accurate modeling of the system. It is very important to examine this category of indeterminacy, especially in the model reference control method, because the basis of the model reference method is model based and is highly dependent on the exact model. To add a nonparametric indeterminacy, since $x_{1}$ is present in most of the equations of the boiler system equation, so we convert the terms from $x_{1}^{9 / 8}$ to $x_{1}^{9 / 8}+0.2 x_{1}^{1 / 8}$. It should be noted that any coefficient and power can be considered and the control system evaluated. Also, with an indefinite magnification, it is observed that the control system has gradually become weaker, and from now on, the system cannot be controlled with a large indeterminacy. Therefore, in this category, any indefinite type can be tested with trial and error. Figure 10 shows the performance of the proposed model reference control system in the boiler output steam pressure in the presence of nonparametric uncertainty.

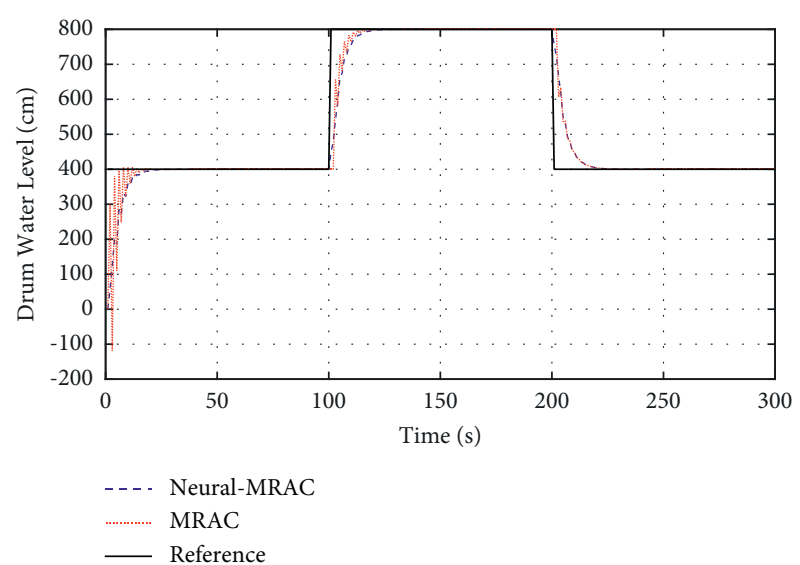

FIGURE 6: Droplet water model reference control.

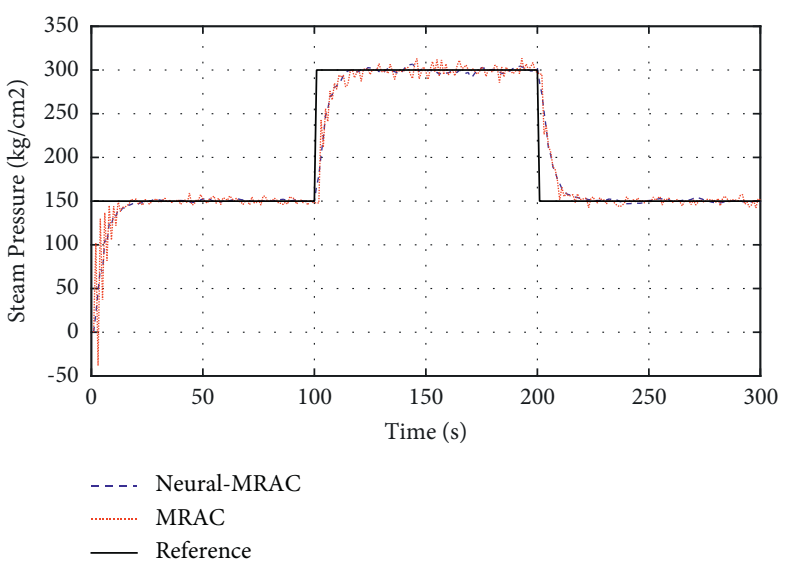

FIGURE 7: Steam pressure control in the presence of an uncertain parameter of $\pm 10 \%$.

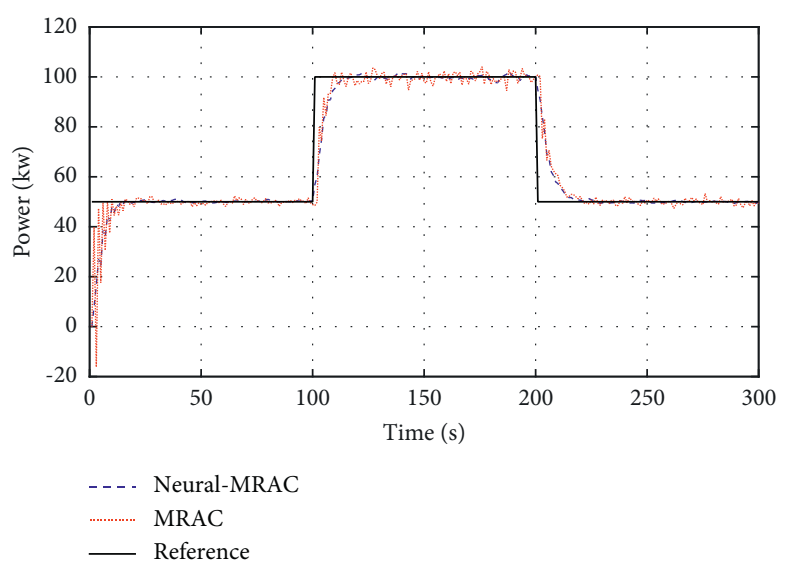

FIGURE 8: Electrical power model reference control in the presence of an uncertain parameter of $\pm 10 \%$.

Figure 11 shows the performance of the model reference control system to control the electric power in the presence of an indefinite nonparametric presence. 


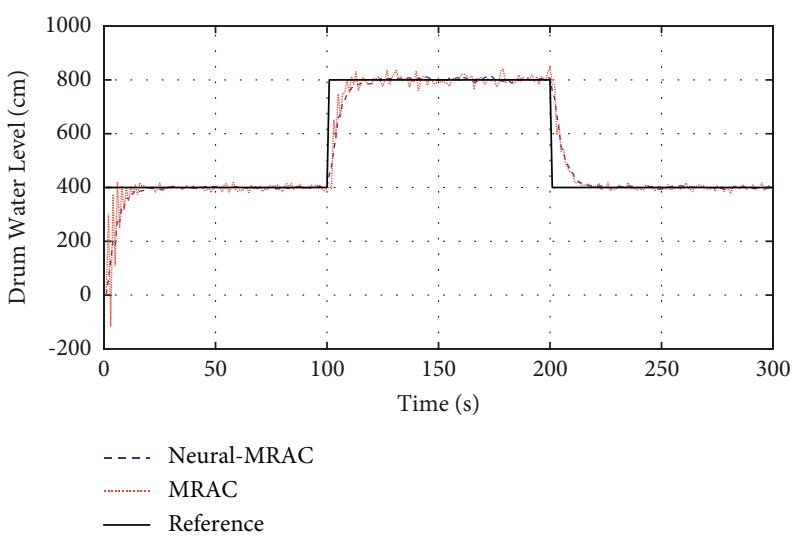

Figure 9: Control of the model reference of the drum water level in the presence of an uncertain parameter of $\pm 10 \%$.

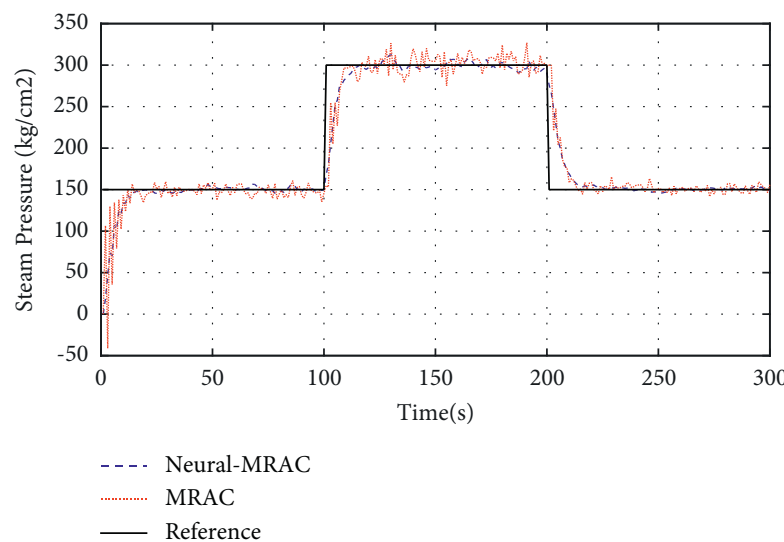

FIGURE 10: Control of the steam pressure model reference in the presence of nonparametric uncertainty.

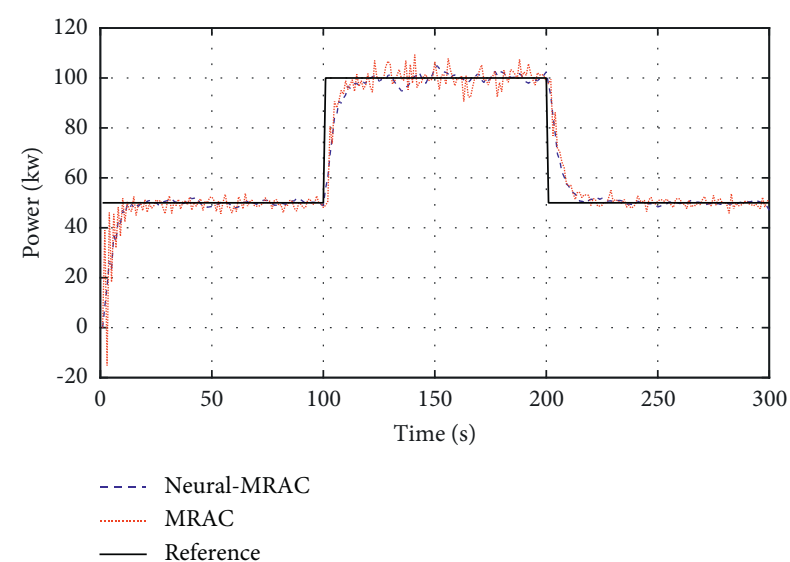

FIGURE 11: Control of the power model reference in the presence of nonparametric uncertainty.

Figure 12 shows the performance of the model reference control system to control the water level of the drum in the presence of nonparametric uncertainty.

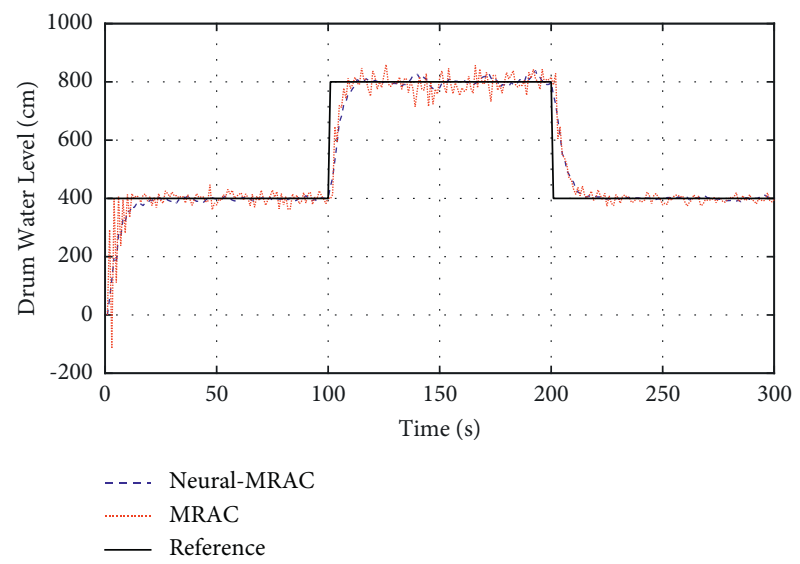

FIgURE 12: Control of the model reference of the drum water level in the presence of nonparametric uncertainty.

As shown in Figure 4 to 12, the use of recurrent type-2 fuzzy RBFN in the model reference control method leads to a significant reduction in fluctuations and control ripples. Also, when the reference signal has a sudden change, the recurrent type- 2 fuzzy RBFN immediately directs the output of the system in the direction of the change and there is no time delay. For further comparison, Table 2 shows the performance of the proposed recurrent type-2 fuzzy neural network (RT2FRBFN) with two methods including normal (without feedback) type-2 fuzzy neural network (T2FRBFN) and type-1 fuzzy neural network (T1FRBFN).

Table 2 shows significant results. First, the number of fuzzy rules in RT2FRBFN is much less than the other two methods, especially, compared with the type-1 fuzzy neural network. Secondly, with an uncertainty increase, the number of fuzzy rules for our proposed model has only increased by 1 , but in other methods, more than 1 rule has been added. The RMSE index also confirms the superiority of our proposed model. However, the training time in our proposed model is longer than the model without feedback, which is normal and related to feedback calculations. 
TABLE 2: Comparison between our proposed method with other two methods.

\begin{tabular}{lcccccr}
\hline & \multicolumn{2}{c}{ Number of fuzzy rules } & \multicolumn{2}{c}{ Root mean squares error } & \multicolumn{2}{c}{ Training time } \\
& Unc. $0 \%$ & Unc. $10 \%$ & Unc. $0 \%$ & Unc. $10 \%$ & Unc. $0 \%$ & Unc. $10 \%$ \\
\hline T1FRBFN & 26 & 41 & 0.275 & 0.344 & 51 & 55 \\
T2FRBFN & 9 & 11 & 0.093 & 0.115 & 33 & 38 \\
RT2FRBFN & 6 & 7 & 0.072 & 0.098 & 38 & 42 \\
\hline
\end{tabular}

\section{Conclusion}

Today, boilers are widely used in the industry. Precise control and mastery of the boilers and their output parameters are very much needed. Since the nature of thermal systems in terms of control is complex systems, therefore, no control method can be used. In this paper, in order to control the boiler, the method of adaptive model reference based on recurrent type- 2 fuzzy RBFN was proposed. The proposed method is resistant to parametric and nonparametric changes, and since the control system depends on the control system, so with the model changes, the control system also changes. Two indefinite parametric and nonparametric models were considered for the system. In the indefinite parameter, it was assumed that the parameters in the system model would change, and in the nonparametric indefiniteness, terms were added to the system model. The results showed that, with about $\pm 10 \%$ increase in the amount of both indeterminate models, the model reference control system provided good capability. However, with the further increase of uncertainties (for example, about $\pm 20 \%$ ), the model reference control system does not function properly. Comparing the conventional model reference method and the recurrent type-2 fuzzy RBFN-based model reference method, it has been observed that the use of recurrent type- 2 fuzzy RBFN leads to a reduction in control ripples as well as a reduction in the time delay of the control system.

\section{Data Availability}

The industrial boiler data used to support the findings of this study are available from the corresponding author upon request.

\section{Conflicts of Interest}

The authors declare that they have no conflicts of interest.

\section{References}

[1] Md. N. Hossain, K. Ghosh, and N. K. Manna, "A multiphase model for determination of minimum circulation ratio of natural circulation boiler for a wide range of pressure," International Journal of Heat and Mass Transfer, vol. 150, 2020.

[2] S. Ghabraei, H. Moradi, and G. Vossoughi, "Design \& application of adaptive variable structure \& Ho robust optimal schemes in nonlinear control of boiler-turbine unit in the presence of various uncertainties," Energy, vol. 142, pp. 1040-1056, 2018.

[3] J. Peng, Z. Cao, X. Yu, Y. Yu, G. Chang, and Z. Wang, "Investigation of flame evolution in heavy oil boiler bench using high-speed planar laser-induced fluorescence imaging," Applied Sciences, vol. 8, no. 9, p. 1691, 2018.

[4] S. Kamerling, V. Vuillerme, and S. Rodat, "Solar field output temperature optimization using a MILP algorithm and a $0 \mathrm{D}$ model in the case of a hybrid concentrated solar thermal power plant for SHIP applications," Energies, vol. 14, no. 13, p. 3731, 2021.

[5] O. Mohamed, A. Khalil, and J. Wang, "Modeling and control of supercritical and ultra-supercritical power plants: a review," Energies, vol. 13, no. 11, p. 2935, 2020.

[6] F. Xia, Y.-L. Quan, H. Zhang, and D.-G. Peng, "Water level deviation analysis of levels between two sides of the boiler drum," International Journal of Automation and Computing, vol. 13, no. 2, pp. 142-150, 2016.

[7] F. M. de Mello, A. J. G. da Cruz, and R. de Sousa, "Fuzzy control applied to combustion in sugarcane bagasse boilers," in Computer Aided Chemical Engineering, A. A. Kiss, Ed., Elsevier, Amsterdam, Netherlands, pp. 1135-1140, 2019.

[8] J.-L. Gao, "Research on boiler water supply control system based on AT89C55 and fractional order PID algorithm," Procedia Computer Science, vol. 154, pp. 173-180, 2019.

[9] S. Darwish, A. Pertew, W. Elhaweet, and A. Mokhtar, "Advanced boiler control system for steam power plants using modern control techniques," in Proceedings of the 2019 IEEE 28th International Symposium on Industrial Electronics (ISIE), Vancouver, Canada, June 2019.

[10] M. H. Toodeshki and J. Askari, "Model-Reference Adaptive Control for a nonlinear boiler-turbine system," in Proceedings of the 2008 IEEE International Conference on Industrial Technology, Chengdu, China, April 2008.

[11] J. Tavoosi, A. A. Suratgar, and M. B. Menhaj, "Stability analysis of a class of MIMO recurrent type-2 fuzzy systems," International Journal of Fuzzy Systems, vol. 19, no. 3, pp. 895-908, 2017.

[12] J. Tavoosi, A. A. Suratgar, and M. B. Menhaj, "Stable ANFIS2 for nonlinear system identification," Neurocomputing, vol. 182, pp. 235-246, 2016.

[13] M. Krawczak and G. Szkatuła, "On matching of intuitionistic fuzzy sets," Information Sciences, vol. 517, pp. 254-274, 2020.

[14] J. Tavoosi and B. MA, "A class of type-2 fuzzy neural networks for nonlinear dynamical system identification," Neural Computing \& Applications, vol. 23, no. 34, pp. 707-717, 2013.

[15] J. Tavoosi, A. A. Suratgar, and M. B. Menhaj, "Stability analysis of recurrent type-2 TSK fuzzy systems with nonlinear consequent part," Neural Computing \& Applications, vol. 28, no. 1, pp. 47-56, 2017.

[16] J. Tavoosi, "An experimental study on inverse adaptive neural fuzzy control for nonlinear systems," International Journal of Knowledge-Based and Intelligent Engineering Systems, 2020, In press.

[17] M. B. B. Sharifian, A. Mirlo, J. Tavoosi, and M. Sabahi, "Selfadaptive RBF neural network PID controller in linear elevator," in Proceedings of the International Conference on Electrical Machines and Systems, Beijing, China, August 2011. 
[18] J. Tavoosi, M. Alaei, and B. Jahani, "Temperature control of water bath by using neuro-fuzzy controller," in Proceedings of the 5th Symposium on Advance in Science and Technology, Mashhad, Iran, May 2011.

[19] B. Hu and J. Wang, "Deep learning based hand gesture recognition and UAV flight controls," International Journal of Automation and Computing, vol. 17, no. 1, pp. 17-29, 2020.

[20] M. Chavoshian, M. Taghizadeh, and M. Mazare, "Hybrid dynamic neural network and PID control of pneumatic artificial muscle using the PSO algorithm," International Journal of Automation and Computing, vol. 17, no. 3, pp. 428-438, 2020.

[21] J. Tavoosi, "A novel recurrent type-2 fuzzy neural network for stepper motor control mechatronic systems and control," vol. 49, no. 1, 2021.

[22] L. Kong, W. He, C. Yang, Z. Li, and C. Sun, "Adaptive fuzzy control for coordinated multiple robots with constraint using impedance learning," IEEE Transactions on Cybernetics, vol. 49, no. 8, pp. 3052-3063, 2019.

[23] W. He, H. Gao, C. Zhou, C. Yang, and Z. Li, "Reinforcement learning control of a flexible two-link manipulator: an experimental investigation," IEEE Transactions on Systems, Man, and Cybernetics: Systems, pp. 1-11. In press, 2020.

[24] Y. P. Asad, A. Shamsi, and J. Tavoosi, "Backstepping-based recurrent type-2 fuzzy sliding mode control for MIMO systems (MEMS triaxial gyroscope case study)," International Journal of Uncertainty, Fuzziness and Knowledge-Based Systems, vol. 25, no. 2, pp. 213-233, 2017.

[25] Y. Pour Asad, A. Shamsi, H. Ivani, and J. Tavoosi, "Adaptive intelligent inverse control of nonlinear systems with regard to sensor noise and parameter uncertainty (magnetic ball levitation system case study)," International Journal on Smart Sensing and Intelligent Systems, vol. 9, no. 1, 2016.

[26] J. Tavoosi, A. Shamsi Jokandan, and M. A. Daneshwar, "A new method for position control of a 2-DOF robot arm using neuro-fuzzy controller," Indian Journal of Science and Technology, vol. 5, no. 3, 2012.

[27] J. Tavoosi, B. Ma, and S. Ghaemi, "Adaptive inverse control of nonlinear dynamical system using type-2 fuzzy neural networks," Journal of Control, vol. 5, no. 2, pp. 52-60, 2011.

[28] W. Yu, F. Zhao, H. Xu et al., "Predictive control of CO2 emissions from a grate boiler based on fuel nature structures using intelligent neural network and Box-Behnken design," Energy Procedia, vol. 158, pp. 364-369, 2019.

[29] F. M. de Mello, A. J. G. da Cruz, R. de Sousa, and R. de Sousa, "Fuzzy control applied to combustion in sugarcane bagasse boilers," in Computer Aided Chemical Engineering, A. A. Kiss, E. Zondervan, R. Lakerveld, and L. Özkan, Eds., vol. 46, pp. 1135-1140, Elsevier, Amsterdam, Netherlands, 2019.

[30] X. Zhuo, C. Lou, H. Zhou, J. Zhuo, and P. Fu, "Hierarchical Takagi-Sugeno fuzzy hyperbolic tangent static model control for a circulating fluidized bed boiler thermal power unit," Energy, vol. 162, pp. 910-917, 2018.

[31] D. Xiu-Cheng, W. Hai-Bin, and Z. Xiao-Xiao, "Model reference neural network control for boiler combustion system," in Proceedings of the 2005 International Conference on Machine Learning and Cybernetics, Guangzhou, China, August 2005.

[32] J.-Q. Li, J.-Z. Liu, Y.-G. Niu, C.-L. Niu, and W. Liu, “Application of neural network model reference adaptive control in coal-fired boiler combustion system," in Proceedings of the 2004 International Conference on Machine Learning and Cybernetics, pp. 564-567, Shanghai, China, August 2004.
[33] K. Xiangsong, C. Xurui, and G. Jiansheng, "PID controller design based on radial basis function neural networks for the steam generator level control," Cybernetics and Information Technologies, vol. 16, no. 5, pp. 15-26, 2016.

[34] S. Yuan, B. De Schutter, and S. Baldi, "Robust adaptive tracking control of uncertain slowly switched linear systems," Nonlinear Analysis: Hybrid Systems, vol. 27, pp. 1-12, 2018.

[35] S. Yuan, B. De Schutter, and S. Baldi, "Adaptive asymptotic tracking control of uncertain time-driven switched linear systems," IEEE Transactions on Automatic Control, vol. 62, no. 11, pp. 5802-5807, 2017.

[36] K. Åström and R. Bell, "Dynamic models for boiler-turbine alternator units: data logs and parameter estimation for a 160 MW unit," Technical Reports (TFRT-3192), 1987.

[37] J. Tavoosi, A. A. Suratgar, and M. B. Menhaj, "Nonlinear system identification based on a self-organizing type-2 fuzzy RBFN," Engineering Applications of Artificial Intelligence, vol. 54, 2016.

[38] B. Vaseghi, M. A. Pourmina, and S. Mobayen, "Finite-time chaos synchronization and its application in wireless sensor networks," Transactions of the Institute of Measurement and Control, vol. 40, no. 13, pp. 3788-3799, 2018.

[39] M. Firouzi, M. Nasiri, S. Mobayen, and G. B. Gharehpetian, "Sliding mode controller-based BFCL for fault ride-through performance enhancement of DFIG-based wind turbines," Complexity, vol. 2020, Article ID 1259539, 12 pages, 2020.

[40] M. Golestani, S. Mobayen, and H. Richter, "Fast robust adaptive tracker for uncertain nonlinear second-order systems with time-varying uncertainties and unknown parameters," International Journal of Adaptive Control and Signal Processing, vol. 32, no. 12, pp. 1764-1781, 2018. 